\title{
La calidad del cuidado de Enfermería y el nivel de satisfacción de las necesidades espirituales en los pacientes del servicio de emergencia del Hospital José Agurto Tello, Lurigancho, Chosica, 2012
}

\author{
Carmen QUINTANA DAMIÁN ${ }^{*}$, Claudio Moisés HUAMANÍ QUINTANA, ${ }^{2}$ \\ ${ }^{1}$ Servicio de Emergencia, Hospital José Agurto Tello, Chosica, Lima \\ ${ }^{2}$ Clinica Adventista Ana Stahl, Av. La Marina 285, Iquitos.
}

\begin{abstract}
RESUMEN
El objetivo del estudio fue determinar la relación que existe entre la calidad del cuidado de enfermería y el nivel de satisfacción de las necesidades espirituales en los pacientes del servicio de emergencia del Hospital José Agurto Tello de Chosica. Estudio cuantitativo, descriptivo correlacional, porque asocia variables mediante un patrón predecible para un grupo o población. De corte trasversal. Se utilizó el muestreo no probabilístico, según criterios de inclusión, los cuales fueron: Pacientes adultos de ambos sexos hospitalizados en el servicio de emergencia y enfermeras que laboran en este servicio, teniendo una muestra de 40 pacientes y 40 enfermeras. Mediante la prueba de independencia chi cuadrado se llegó a la conclusión que no existe relación de asociación entre la calidad del cuidado de enfermería y el nivel de satisfacción de las necesidades espirituales de los pacientes en términos de (a) sentido de pertenencia, amor y respeto, (b) lo divino, (c) positividad, gratitud, esperanza y paz, (d) significado y objetivo de la vida, (e) moralidad y ética, (f) reconocimiento de la belleza y (g) resolución ante la muerte. Llegando a la conclusión que cuanto más eficiente es la calidad del cuidado del profesional de enfermería no influye en nivel de satisfacción de las necesidades espirituales de los pacientes. Lo que no significa que el profesional de enfermería debe brindar calidad del cuidado espiritual hacia el paciente.
\end{abstract}

Palabras clave: Calidad, cuidado de enfermería, satisfacción, necesidades espirituales.

\begin{abstract}
The aim of the study was to determine the relationship between the quality of nursing care and the level of satisfaction of the spiritual needs of patients in the Emergency Department of Hospital José Agurto Chosica Tello. Quantitative study, descriptive correlational because variables associated with a predictable pattern for a group or population. Cross cutting. We used non-probability sampling, as inclusion criteria, which were: adult male and female patients hospitalized in the emergency room and nurses working in the emergency room, taking a sample of 40 patients and 40 nurses. By chi square test of independence is concluded that there is no relationship of partnership between the quality of nursing care and the level of satisfaction of the spiritual needs of patients in terms of (a) sense of belonging, love and respect , (b) the Divine, (c) positivity, gratitude, hope and peace, (d) the meaning and purpose of life, (e) morality and ethics, (f) recognition of beauty and (g) resolution before death . Concluding the more efficient is the quality of care the nurse does not influence the level of satisfaction of patients' spiritual needs. This does not mean that the nurse should provide quality spiritual care to the patient.
\end{abstract}

Keywords: Quality, nursing care, satisfaction, spiritual needs.

*Correspondencia: carmenquintana_1@hotmail.com 


\section{INTRODUCCIÓN}

El abordaje para trabajar esta temática se hizo con la medición y comparación del bienestar espiritual evidenciado por la presencia del significado, propósito y plenitud en la vida, el deseo de vivir, creer y tener fe en el propio ser, y en Dios, porque en él se ve la totalidad del ser humano cuya naturaleza corporal y espiritual están unidas y son inesperables.

Asimismo, las necesidades espirituales son el vigor natural y la virtud que alienta y fortifica, da fuerza y valor para afrontar los acontecimientos de la salud, por esta razón el personal debe estar capacitado no solo en el campo clínico sino también en el aspecto espiritual, permitiendo brindar un cuidado holístico, situación que el paciente espera ser satisfecha. (Suarez, 1998).

El cuidado de enfermería también debe basarse en la satisfacción de las necesidades espirituales del paciente para la cual va a determinar procedimientos necesarios y las necesidades de enseñanza de salud. (Carrvitero, 2008).

La organización mundial de la salud reconoció por primera vez al cuidado espiritual como valor y dimensión básica de la calidad de cuidado de enfermería, estableciendo el vínculo que constituye la clave para una comprensión holística de la salud (Maceiras, 2011).

El cuidado espiritual es importante y se manifiesta a través de la oración, la meditación, la lectura de la biblia y las creencias en la vida eterna, sensibilidad humana, la bondad, la solidaridad, la honestidad, el desinterés material, el trato afectuoso indiscriminado, la lealtad, y la disposición al esfuerzo y sacrificio mostrando su calidad de vida espiritual que posee, lo refiere (Águila 2011). Entonces garantizar la calidad exige a los profesionales de enfermería una reflexión permanente sobre valores, actitudes, aptitudes y normas que orientan los objetivos del bien interno, mediante la implementación de estrategias que privilegien "cuidar con calidad” y para hablar de calidad en salud es esencial hacer conciencia sobre la diversidad étnica y cultural del país, por ello más que un esfuerzo de homogenización, es básico un enfoque de diálogo intercultural, tanto en la definición de objetivos de calidad como en las acciones concretas de mejoramiento (Colegio de Enfermeros del Perú, 2008).

Evidentemente cuando se lee o se escucha la palabra satisfacción se tiene en cuenta lo difícil que resulta para el profesional de enfermería cubrir las expectativas que el usuario trae consigo, ya lo dijo Martha Rogers (1945), la expectativa de cada usuario es única y está también sujeta a cambios durante el proceso de su estancia en la institución de salud (Potter, 2011).

La espiritualidad es esencial en la naturaleza humana y es el recurso más profundo y potente de sanación que tiene la persona (Álvarez, 2009).

Las necesidades espirituales y el mantenimiento del bienestar espiritual son componentes importantes en el ser humano, pero con frecuencia es un aspecto olvidado y no ofrecido en el cuidado holístico que se le ofrece al paciente, las creencias religiosas o espirituales asumen gran importancia en el momento de la enfermedad y en momentos trascendentales en la vida de la persona (Suárez, 1998).

En la revisión bibliográfica acerca del papel que tiene la Espiritualidad sobre la salud, se evidenció cómo la espiritualidad puede convertirse en una poderosa fuente de fortaleza, ya que capacita al individuo a hacer cambios positivos en su estilo de vida y a tomar conciencia de cómo las creencias, actitudes y comportamientos pueden afectar positiva o negativamente su salud. Se evidencia, en este trabajo, cómo las prácticas religiosas pueden ayudar a las personas a sobrellevar el estrés del día a día y, especialmente, a aquellas sometidas al estrés agudo. Por otro lado, debemos conocer que la religiosidad y la espiritualidad, como estrategias de afrontamiento, parecen estar asociadas a una mejor salud mental y a una rápida adaptación al estrés, particularmente, al estrés prolongado. Se demuestra ampliamente, en la literatura revisada, cómo al promover el bienestar existencial del individuo, ya sea a través de sus creencias religiosas o sus prácticas espirituales, se favorece una mejor función del sistema inmunológico, toda vez que la espiritualidad favorece un estilo de vida diferente y permite que nuestros comportamientos sean más sanos, y esto se asocia a un menor riesgo de enfermedades y a una actitud diferente (Navas, 2006).

Las enfermeras han asumido la responsabilidad de brindar al usuario los cuidados físicos necesarios para su restablecimiento, representado en múltiples situaciones; igualmente le proporciona información útil para fomentar hábitos saludables en la vida cotidiana y así como también la prevención de enfermedades, en este sentido para que el usuario deposite su confianza en la enfermera, está debe saber cuál es el alcance de la responsabilidad de la misma (Dugas, 2000).

En el proceso de la satisfacción de las necesidades de los pacientes asignados al cuidado, el enfermero recurre a una relación de cooperación y participación activa que se debe enfocar no solo al cuidado de lo biológico, sino también al cuidado espiritual, para producir los resultados esperados, en relación con la recuperación del bienestar o condicionar una muerte tranquila, con un enfoque holístico. Equivale a decir que los enfermeros no solo deben abocarse a la corrección de la insatisfacción de las necesidades físicas, sino que se deben 
identificar e intervenir en aquellas situaciones de tipo espiritual y social: deben cuidar no solo el cuerpo, sino también la persona y el espíritu, debido a que los sentimientos de frustración, conflicto y ansiedad, estados de estrés que permanecieran sin resolver, podrán contribuir a la inestabilidad física y al retraso en el proceso de mejoría y curación (Navas, 2006).

El personal de enfermería está capacitado para brindar atención integral en forma oportuna, segura, continua y humanística; según Blocker (1995), dicha atención al ser experimentada por el paciente, permitirá determinar a través de su opinión la calidad y calidez de los cuidados de enfermería que ha recibido durante su hospitalización.

Dentro de este orden de ideas, el objeto del estudio fue determinar la relación que existe entre la calidad del cuidado de enfermería y el nivel de satisfacción de las necesidades espirituales en los pacientes del servicio de emergencia del Hospital José Agurto Tello de Chosica.

\section{MATERIAL Y MÉTODOS}

Estudio cuantitativo, descriptivo correlacional, porque asocia variables mediante un patrón predecible para un grupo o población. De corte trasversal porque se realizó la investigación de marzo a diciembre del 2011. Se utilizó el muestreo no probabilístico, según criterios de inclusión, los cuales fueron: Pacientes adultos de ambos sexos hospitalizados en el servicio de emergencia y Enfermeras profesionales que laboran en el servicio de emergencia, teniendo una muestra de 40 pacientes y 40 enfermeras. Se elaboró un cuestionario que midió la calidad del cuidado de enfermería y el nivel de satisfacción de las necesidades espirituales del paciente en emergencia, este cuestionario tuvo dos partes: la primera que está dirigida a los profesionales de enfermería con la variable calidad del cuidado de enfermería con sus dimensiones de evaluación e implementación de la atención espiritual, profesionalización y el cuidado espiritual, apoyo y consejería al paciente, referencia a los profesionales, actitud hacia la espiritualidad del paciente, comunicación. La segunda variable está dirigiga al nivel de satisfacción de las necesidades espirituales, y sus dimensión: amor, pertenencia y respeto, lo divino, positividad y gratitud, esperanza y paz, significado y propósito, moral y ética, reconocimiento de belleza, resolución/muerte. El cuestionario tuvo 30 preguntas, cuyas respuestas corresponde a la escala de Likert. Los instrumentos fueron validados por juicios de expertos que acreditan la experiencia en el área, y respecto a la validez interna se realizó con el alpha de Crombach, obteniendo para el instrumento de calidad del cuidado de enfermería 0.646 y para el instrumento de necesidades espirituales 0.852 , indicando el grado de coherencia interna del instrumento.

La encuesta se tomó en cada turno de los profesionales que laboraban en el servicio de emergencia y a los pacientes se les tomó cada mañana, previo consentimiento informado.

Para el análisis de datos, se uso la prueba del Chi cuadrado.

\section{RESULTADOS}

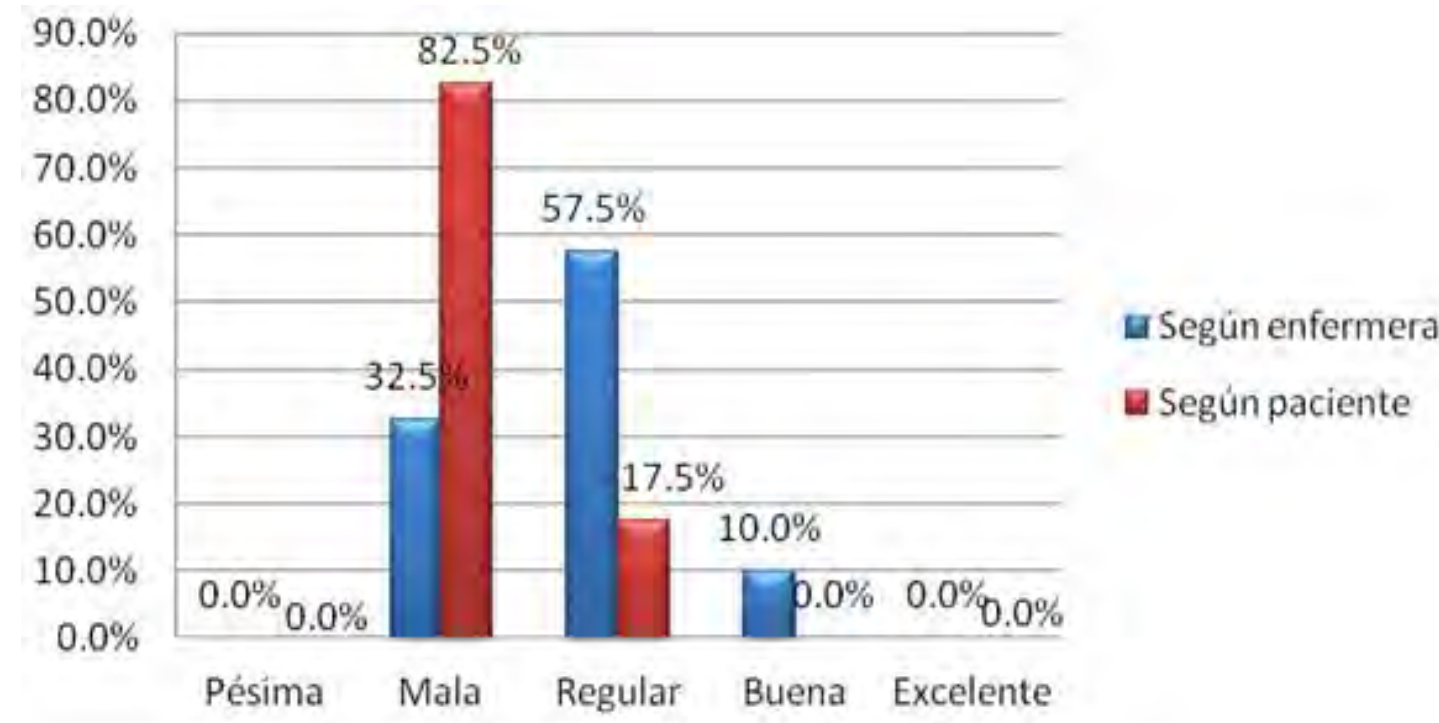

\section{Figura 1.}

Comparación entre la percepción que tiene el profesional de enfermería y el paciente sobre la calidad de los cuidados en la satisfacción de las necesidades espirituales. 
Según la figura 1, el $82.5 \%$ de pacientes percibe un nivel malo respecto a la calidad del cuidado que brinda el profesional de enfermería en la satisfacción de sus necesidades espirituales, constrantando con la opinión del profesional de enfermería, donde el 57.5\% lo percibe regular, y un 10\% lo percibe bueno.

\section{Tabla 1}

Prueba Chi-cuadrado de Pearson de la calidad del cuidado de enfermería y el nivel de satisfacción de las necesidades espirituales del paciente.

\begin{tabular}{ccccc}
\hline Chi-cuadrado de Pearson & Valor & gl & $\begin{array}{c}\text { Sig. asintótica } \\
\text { (bilateral) }\end{array}$ & Conclusión \\
\hline $\begin{array}{c}\text { Necesidades espirituales en sentido de } \\
\text { amor, pertenencia y respeto }\end{array}$ & 0.803 & 2 & 0.669 & No se rechaza la Ho. \\
$\begin{array}{c}\text { Necesidades espirituales en lo divino } \\
\begin{array}{c}\text { Necesidades espirituales en positividad, } \\
\text { gratitud, esperanza y paz }\end{array}\end{array}$ & 2.534 & 3 & 0.469 & No se rechaza la Ho. \\
$\begin{array}{c}\text { Necesidades espirituales en significado } \\
\text { y objetivo }\end{array}$ & 2.039 & 3 & 0.792 & No se rechaza la Ho. \\
$\begin{array}{c}\text { Necesidades espirituales en moralidad } \\
\text { y ética }\end{array}$ & 2.771 & 3 & 0.52 & No se rechaza la Ho. \\
$\quad \begin{array}{c}\text { Necesidades espirituales en } \\
\text { reconocimiento de la belleza }\end{array}$ & 1.348 & 3 & 0.718 & No se rechaza la Ho. \\
$\begin{array}{c}\text { Necesidades espirituales en resolución } \\
\text { ante la muerte }\end{array}$ & 1.8 & 3 & 0.615 & No se rechaza la Ho. \\
\hline
\end{tabular}

Si p-value (Sig. asintótica bilateral) $\geq \alpha=0.05$, la prueba no es significativa, no se rechaza la Ho.

Si p-value (Sig. asintótica bilateral) $<\alpha=0.05$, la prueba es significativa, se rechaza la Ho.

No existe relación de asociación entre calidad del cuidado de enfermería y nivel de satisfacción de las necesidades espirituales en los pacientes del servicio de emergencia del Hospital José Agurto Tello de Chosica. Significa que cuanto más eficiente es la calidad del cuidado del profesional de enfermería no influye en nivel de satisfacción de las necesidades espirituales de los pacientes. Lo que no significa que el profesional de enfermería debe brindar calidad del cuidado espiritual hacia el paciente.

\section{DISCUSIÓN}

Uno de los grandes retos de enfermería es demostrar la efectividad del cuidado del paciente, en este sentido hablar de calidad del trabajo de enfermería, teniendo en cuenta la opinión del propio personal, es de gran importancia, debido a que de alguna manera los cuestionamientos sobre esto llevan a la autorreflexión del quehacer profesional, además representa una oportunidad de autovaloración, lo que permite tener la apreciación de quienes con frecuencia son sujetos de valoración.

El análisis de necesidades espirituales reveló elementos que reflejan las preocupaciones del paciente relativas a la espiritualidad: Amor y pertenencia /respeto; divino, positividad/gratitud/esperanza/paz; significado, resolución /muerte.

Al comparar los resultados de la percepción que tiene el profesional de enfermería y el paciente sobre la calidad de los cuidados en la satisfacción de las necesidades espirituales, se puede observar que presentan diferencias significativas, lo que confirma la prueba estadística, que indica que no existe relación entre ambas variables.

Los resultados obtenidos tienen relación con lo descrito por Martínez (2010), en el artículo "Prospectiva de la enfermera en México” donde relaciona la estructura, proceso y resultado, indicando que el proceso son las acciones que se llevan a cabo por el personal de enfermería, interactuando con el usuario y con otro profesionales de la salud. En otras palabras durante el proceso, en el caso de la práctica de enfermería, se refiere a la planeación y ejecución, intervenciones donde el profesional establece una relación y confianza necesaria y primordial para aplicar con éxito los conocimientos y las habilidades que permitan satisfacer las necesidades del usuario desde la integralidad. 
La ausencia de relación significativa entre las variables de estudio podría ser porque la calidad del cuidado incluye varios procesos y etapas. Así se observó en un estudio realizado en el Hospital II EsSalud de Piura (2009), que tuvo como objetivo determinar la calidad del cuidado enfermero, siendo los resultados en las dimensiones científico-técnico con el $80.13 \%$ interpersonal $69.31 \%$ y de confort 67.31 . Estos resultados concuerdan con los obtenidos por Ascope y Guzmán (2010), que dentro de su investigación consideran a la dimensión científico-técnica dando como resultado $49 \%$ y $45 \%$ respectivamente, calificando como eficiente la calidad del cuidado de enfermería, mientras que a la dimensión interpersonal el $58 \%$ calificó como bueno y el componte de confort el $51.7 \%$ de los usuarios adultos califica al entorno hospitalario como eficiente y el $74 \%$ como no eficiente.

Por otro lado, el profesional de enfermería está capacitado para brindar cuidado integral y holístico al usuario, dentro de éste el de brindar un cuidado espiritual. Asimismo, en la tarea de cuidar, principalmente en el plano de la relación de ayuda, es importante estar receptivos a todos estos elementos que son fuente de comunicación de la recepción individual y subjetiva de la persona enferma y necesitada de ayuda, y esto es así, porque la enfermedad, estudiada desde el paradigma biomédico, tiene sus manifestaciones y experiencias físicas y fisiológicas, pero, desde un enfoque antropológico, afecta el código subjetivo del ser humano, a su interioridad y a su mundo emotivo y relacional (Puchalski 2006).

En ese contexto, se ha definido el cuidado espiritual como hacer diversas intervenciones para satisfacer las necesidades espirituales de la persona cuidada, lo que incluye ayudar a identificar y desarrollar su perspectiva espiritual y su conciencia personal de espiritualidad y de sus componentes (Sánchez, 2004).
En el estudio, dentro de las dimensiones de la variable necesidades, resalta el resultado de $82.5 \%$ de los pacientes se encuentran insatisfechos de la necesidad espiritual en amor, pertenecía y respeto. Cabe mencionar que este dato debe ser una oportunidad para mejorar el cuidado espiritual que brinda el profesional de enfermería.

Al respecto, Ordoñez (2006), indica que la relación y comunicación se establece a través de la realización de procedimientos, mas no a través de sus pensamientos o su sentir, pues ello no evidencia objetivamente. Por lo cual el cuidado que se debe brindar debe ser holístico, desarrollando una asistencia humanizada, autentica buscando el encuentro de sí mismo, ayudándolos a madurar y encontrar el sentido de la vida, su vocación en el mundo.

Cabe mencionar que la influencia del cuidado espiritual es de importancia en la recuperación de los pacientes en situaciones críticas como es el caso de los participantes del estudio, por estar en situaciones de emergencia, lo que corrobora el trabajo realizado por Bullón y Gabino (2009), en el Hospital Nacional Guillermo Almenara Irigoyen, para determinar la influencia del cuidado espiritual que brinda el profesional de enfermería en el estado de ánimo, nivel de colaboración y signos vitales de los pacientes hospitalizados en la Unidad de Cuidados Intensivos. El diseño del estudio fue cuantitativo, de tipo cuasi experimental, con grupo control y experimental. Llegando a la conclusión que el cuidado espiritual que brinda el profesional de enfermería influye positivamente en el estado de ánimo y nivel de colaboración de los pacientes de la UCI con un nivel de significancia de 0.000 para el grupo experimental. Sin embargo, no tiene influencia en las funciones vitales de los pacientes. 


\section{REFERENCIAS BIBLIOGRÁFICAS}

1. Águila, N. (2011). La Bioética :Un reto para el crecimiento espiritual de la enfermeria. Recuperdo de http://www.calleb.cult.cu/editorial/2011/narciso_2011.html.

2. Alarcon, I. (2009). Necesidades espirituales de los pacientes con cáncer terminal del servicio de medicina en el hospital Dos de Mayo. Tesis para optar el título de licenciado en enfermeria. Universidad Mayor de San Marcos, Lima Perú.

3. Benito, E. (2009). El sufrimiento al final de la vida. Grupo de trabajo sobre espiritualidad, SECPAL, Mecina Paliativa. pp15:225-238. Recuperado de http://www.slideshare.net/sanidadyconsumo/elsufrimiento-al-final-de-la-vida.

4. Bullón, G., \& Gabino, M.(2009). Influencia del cuidado espiritual que brinda el profesional de enfermería en el estado de ánimo, nivel de colaboración y signos vitales de los pacientes hospitalizados en la Unidad de Cuidados Intensivos del Hospital Nacional Guillermo Almenara Irigoyen EsSalud. Revista Científica de Ciencias de la Salud, Universidad Peruana Unión; 2010 p.40-47.

5. Dugas, B. Tratado de enfermería práctica. Rela- ción Enfermería-paciente. 4ta Edición MadridEspaña: Mc Graw - Hill Interamericana; 2000pp 117-118.

6. Martinez, P. (2005). Calidad de atencion en la unidd de cuidados intensivos 2C, Hospital Nacional Edgardo Rebagliati Martins. Essalud.

7. Navas, C. (2006). Espiritualidad y salud. Revista de la ciencia de la educación p.40.Año 6 Vol.1N 27 Valencia. Recuperado de http://servicio.bc.uc. edu.ve/educacion/revista/volIn .27/27-2.pdf.

8. Potter y Perry. (2011). Fundamento de Enfermería, teoría-Práctica. 2Vol 5ta Edición. España. pp. 332-334.

9. Puchalski. (2006). La Espiritualidad y la consejería profesional: Enfoque holístico de salud mental. Recuperado de www.scribd.com/.../monografíaLa-Espiritualidad-dentro-d.

10. Sánchez, H. (2004). Dimensión espiritual del cuidado de enfermería en situación de cronicidad y muerte. Bogotá. Recuperado de http://www.bdigital.unal.edu.co/2050/2/beatrizsachezherrera.2004. pdf 